\title{
Significados sobre bioética que emergen en los pacientes participantes en los procesos de formación médica. Medellín-Colombia, 2017
}

\author{
Elsa María Villegas-Múnera', Martha Lucía Escobar-Pérez², Carlos Enrique Yepes-Delgado ${ }^{3}$ \\ David Arnovis Hernández-Carmona ${ }^{4}$
}

\section{RESUMEN}

Objetivo: comprender los significados sobre bioética que emeroen entre los pacientes atendidos en las prácticas académicas en los procesos de formación médica de pregrado y posogrado en Medellín en 2017.

Materiales y métodos: se realizaron 17 entrevistas semiestructuradas en mayores de edad, con un enfoque hermenéutico basado en el paradiogma constructivista. El análisis se efectuó con el uso de técnicas de la teoría fundamentada.

Resultados: como significado central emergió la instrumentalización del paciente cuando participa en el proceso de aprendizaje de los estudiantes, perciben que prima el interés formativo sobre la atención. Otros significados que surgieron son: la subordinación jerárquica en las relaciones clínicas y académicas, la limitación del reconocimiento de derechos y deberes, así como diversidad en la satisfacción con la atención; se resalta su aporte al aprenđizaje, pero expresaron molestia cuando se sentían iognorados en la comunicación docente-estudiantes.

Discusión y conclusión: existe una disposición favorable de los pacientes para participar en la formación médica en procesos no invasivos, pero con limitación del número de estudiantes y con un docente responsable de la atención. La formación médica debe otorgar al paciente un papel activo en las prácticas académicas, reconocer sus necesidades de diálogo, confidencialidad,

1 Docente titular, Facultad de Medicina, Universidad de Antioquia, Medellín, Colombia. Doctora en Bioética.

2 Docente titular, Facultad de Medicina, Universidad de Antioquia, Medellín, Colombia. Magister Salud Colectiva.

3 Docente titular, Facultad de Medicina, Universidad de Antioquia, Medellín, Colombia. Doctor en Epidemiología. Epidemiólogo Hospital Pablo Tobón Uribe.

4 Docente ocasional Facultad Nacional de Salud Pública, Universidad de Antioquia, Medellín, Colombia. Magister Salud Colectiva.

Correspondencia: Martha Lucía Escobar Pérez; martha.escobar@udea.edu.co

Recibido: septiembre 25 de 2018

Aceptado: febrero 5 de 2019

Cómo citar: Villegas-Múnera EM, Escobar-Pérez ML, Yepes-Delgado CE, Hernández-Carmona DA. Significados sobre bioética que emergen en los pacientes participantes en los procesos de formación médica. Medellín-Colombia, 2017. latreia. 20190ct-Dic;32(4):276-287. DOl 10.17533/udea.iatreia.27. 
intimidad, respeto a su autonomía y dignidad, promoviendo relaciones de ellos con estudiantes y profesores basados en valores y principios bioéticos.

\section{PALABRAS CLAVE}

Bioética; Capacitación Profesional; Derechos del Paciente; Educación Médica; Estudiantes de Medicina; Prácticas Clínicas

\section{SUMMARY}

Meanings about bioethics that emerge in patients who had participated in medical training processes. Medellin, Colombia. 2017

Objective: To understand the meanings about bioethics that emerge among patients treated in academic practices, in undergraduate and postoraduate medical training processes in Medellín, 2017.

Materials and methods: With a hermeneutic approach based on the constructivist paradiom, 17 semistructured interviews were conducted in adults. The analysis was carried out with the use of grounded theory techniques.

Results: As a central meaning, the instrumentalization of the patient emerged in the learning process of the students, in which the formative interest over the patient's care prevails. Other meanings that emerged are: hierarchical subordination in clinical and academic relationships, limitation of recognition of rights and duties of patients, as well as diversity in satisfaction with care. There was discomfort when the patient felt ignored in the teaching communication - students. The contribution of the patient to learning is highlighted.

Discussion and conclusion: There is a favorable disposition of the patients to participate in the medical training in non-invasive processes, with limitation of the number of students and with a teacher who is aware of the patient. Medical training should give the patient an active role in academic practices, recognize their needs for dialogue, confidentiality, privacy, respect for their autonomy and dignity, promoting relationships between patients, students and teachers based on bioethical values and principles.

\section{KEY WORDS}

Bioethics; Clinical Clerkship; Medical, Education; Patient Rights; ProfessionaI Training; Students, Medical

\section{INTRODUCCIÓN}

La bioética ha aportado una mirada amplia, procedimental, pluralista, dialógica, inter y transdiciplinaria sobre los asuntos éticos que se desencadenan en todos los seres vivos, en una sociedad cruzada por el avance de la biotecnología y las ciencias de la vida ${ }^{(1 .}$ 2). En el campo de la medicina, la bioética se ha ocupado de los pacientes para superar la concepción centrada en el médico ${ }^{(3)}$. Cuando los pacientes entran en relación con los estudiantes de medicina de pre y posorado, surgen significados sobre las representaciones morales que se producen en el contexto formativo al reconocerlos como agentes activos de los procesos formativos y actores de los currículos médicos ${ }^{(4)}$, cuyo develamiento puede aportar al fortalecimiento de la dimensión bioética en el currículo médico.

La educación médica debe integrar una dimensión holística de los pacientes, puesto que el médico, más allá de atender eventos clínicos y biológicos, se encuentra con seres humanos que tienen expectativas de relaciones de reconocimiento y respeto. En el contexto de sociedades que exaltan el desarrollo tecnológico y científico, la formación de los profesionales de la salud debe basarse en principios y valores bioéticos que dignifiquen los pacientes para evitar que sean tratados de manera insensible. Como lo expresó Sánchez: "las humanidades médicas tienen que abarcar todo lo que nos hace humanos y todo lo que mejora nuestra humanidad" ${ }^{(5)}$.

Por otra parte, hay que destacar lo que señala Molina NP ${ }^{\left({ }^{6}\right)}$ : "Aunque la definición de los ejes temáticos de los cursos de bioética es muy importante, los objetivos de aprendizaje de estos deben ir más allá de los conocimientos teóricos básicos y contemplar los presupuestos necesarios para la adquisición de habilidades como la reflexión, la capacidad crítica y la toma de decisiones, aspectos que se ponen en juego en el ámbito clínico y en la vida cotidiana”.

Para fortalecer la dimensión ética en la formación médica, se requiere integrar al paciente como persona con capacidad de agencia, ello implica hacer 
esfuerzos por investigar su visión sobre los procesos educativos en que intervienen.

Para este trabajo se partió de la pregunta ¿cuáles son los siognificados sobre bioética que emergen entre los pacientes atendidos en el marco de los procesos de formación médica en Medellín? El significado se comprende en este artículo en sintonía con el concepto de Kenneth Gergen, recogido por Arcila Mendoza P.A. et $a I^{(7)}$ : "[Gergen] Interpreta los significados como una construcción relacional que necesita de acciones y suplementos para ser contextualizada; esto quiere decir que el lenguaje no tiene significado por sí solo, tan sólo adquiere su valor en la relación”.

\section{METODOLOGÍA}

La perspectiva epistemológica del presente trabajo es el paradigma constructivista ${ }^{(8)}$, en el cual los datos se asumen como una construcción (en lugar de ser un descubrimiento) mediante la interacción entre investigador y participantes, las valoraciones no se conciben separadas de los hechos. Los datos emergen de situaciones bajo condiciones particulares, de modo que estas condiciones afectan el análisis, ya que objeto y sujeto de investigación son inseparables. De esta forma, el interés de este enfoque es comprender los significados que las cosas tienen para los sujetos que interactúan en el fenómeno investigado. Para explorar la construcción de siognificado que realizan los sujetos, rescata elementos simbólicos como el lenguaje, los gestos, los silencios, los símbolos.

En este modelo se reconocen las múltiples realidades de interpretación y son incorporadas en los análisis: la subjetividad del investigado, la del investigador y la intersubjetividad de ambos. En esta forma de hacer y pensar la investigación, el tiempo de permanencia en el campo y la sensibilidad teórica son fundamentales.

Se utilizó el método de la Teoría fundamentada (TF) desarrollado por Glaser y Strauss ${ }^{(9)}$, el cual se refiere a una progresiva aproximación teórica que emerge del análisis de relatos recopilados de manera sistemática, contempla la codificación como forma de abstracción, pues no solo clasifica o describe fenómenos, sino que permite desarrollar conceptos teóricos que le imprime cierto carácter explicativo de los fenómenos. De otro lado, usa el muestreo teórico una vez empiezan a emerger las categorías iniciales, con lo que se generan los primeros memos analíticos y se guía la elección de los siguientes entrevistados y, por último, se hacen comparaciones teóricas de los contextos y fenómenos estudiados. De esta manera, Ios informantes, quienes son los que viven la experiencia estudiada, expresan su propia visión y perspectiva de lo vivido, van proporcionando información para la construcción que los investigadores hacen de las categorías de la investigación. El análisis fue orientado mediante el proceso de codificación y de categorización, que consiste en momentos iterativos de codificación abierta y axial ${ }^{(9)}$.

El soporte teórico de la TF es el Interaccionismo Simbólico (IS) ${ }^{(10)}$, el cual como corriente sociológica del pensamiento, permite conocer la sociedad o el sujeto de comunicación a partir de las interacciones de los seres humanos con diferentes símbolos para construir significados que les brindan información para entender sus experiencias y las de los demás. Esos significados surgen de la interacción con otros y suelen ser modificados por la interpretación que la persona hace de ellos.

Con previa aprobación del Comité de Bioética de la Facultad de Medicina de la Universidad de Antioquia, se realizaron 17 entrevistas semiestructuradas entre Ios años 2016 y 2017 en Medellín, a personas mayores de edad, atendidas en las prácticas académicas de estudiantes de medicina de pregrado y posgrado de diferentes universidades de la ciudad, en servicios ambulatorios o de hospitalización (Tabla 1). Luego de explicarles el consentimiento informado, los entrevistados permitieron la grabación de la entrevista. La confidencialidad se aseguró asignando a cada entrevistado un código conocido solo por los investigadores y la fiabilidad de la información se garantizó con la transcripción fiel de las entrevistas.

Luego de la codificación abierta de las entrevistas, la codificación axial permitió identificar un fenómeno principal y, relacionado con él, los elementos propios de la matriz paradiogmática ${ }^{(9)}$, definidos como el contexto, las causas, las consecuencias y las relaciones de acción-interacción. Se realizó triangulación entre fuentes de datos y miradas de los investigadores, códigos iniciales y relatos de las entrevistas, códigos y las categorías identificadas y entre la abstracción emergente y la revisión de la literatura científica encontrada 
Tabla 1. Características de los pacientes atendidos con la participación de estudiantes

\begin{tabular}{|c|c|c|c|c|c|c|c|c|}
\hline $\begin{array}{c}\mathrm{N}^{\circ} \\
\text { Entrevista }\end{array}$ & $\begin{array}{c}\text { Edad } \\
\text { paciente }\end{array}$ & Género & $\begin{array}{l}\text { Carácter } \\
\text { institución } \\
\text { prestadora } \\
\text { servicios de } \\
\text { salud (IPS) }\end{array}$ & $\begin{array}{l}\text { Servicio } \\
\text { que prestó la } \\
\text { Atención }\end{array}$ & Especialidad & $\begin{array}{c}\text { Nivel de } \\
\text { Complejidad } \\
\text { IPS }\end{array}$ & $\begin{array}{c}\text { Participación } \\
\text { de estudiantes } \\
\text { pregrado }\end{array}$ & $\begin{array}{c}\text { Participación } \\
\text { estudiantes } \\
\text { posgrado }\end{array}$ \\
\hline 1 & 54 & Femenino & Mixta & $\begin{array}{l}\text { Consulta } \\
\text { externa }\end{array}$ & Dermatología & $\|$ & No & Sí \\
\hline 2 & 46 & Femenino & Privada & $\begin{array}{l}\text { Urgencias } \\
\text { y consulta } \\
\text { externa }\end{array}$ & Neurocirugía & III & Sí & Sí \\
\hline 3 & 27 & Masculino & Pública & $\begin{array}{c}\text { Hospitalización } \\
\text { en urgencias }\end{array}$ & Cirugía & $\|$ & Sí & Sí \\
\hline 4 & 38 & Femenino & Privada & Hospitalización & Pediatría & III & No & Sí \\
\hline 5 & 45 & Femenino & Mixta & $\begin{array}{l}\text { Hospitalización } \\
\text { y consulta } \\
\text { externa }\end{array}$ & Ginecología & $\|$ & Sí & Sí \\
\hline 6 & 40 & Femenino & Privada & Hospitalización & Obstetricia & IV & No & Sí \\
\hline 7 & 52 & Femenino & Privada & Hospitalización & $\begin{array}{l}\text { Medicina } \\
\text { interna }\end{array}$ & III & No & Sí \\
\hline 8 & 56 & Femenino & Mixta & $\begin{array}{l}\text { Consulta } \\
\text { externa }\end{array}$ & Ginecología & $\|$ & No & Sí \\
\hline 9 & 61 & Femenino & Mixta & $\begin{array}{l}\text { Consulta } \\
\text { externa }\end{array}$ & Oftalmología & ॥ & Sí & Sí \\
\hline 10 & 29 & Femenino & Mixta & $\begin{array}{l}\text { Consulta } \\
\text { externa }\end{array}$ & Ginecología & $\|$ & Sí & Sí \\
\hline 11 & 60 & Femenino & Privada & Hospitalización & Psiquiatría & $\|$ & No & Sí \\
\hline 12 & 48 & Femenino & Mixta & $\begin{array}{l}\text { Consulta } \\
\text { externa }\end{array}$ & Fisiatría & $\|$ & No & Sí \\
\hline 13 & 23 & Femenino & Privada & $\begin{array}{l}\text { Consulta } \\
\text { Externa }\end{array}$ & Ortopedia & IV & Sí & Sí \\
\hline 14 & 19 & Femenino & Privada & $\begin{array}{l}\text { Consulta } \\
\text { urgencias y } \\
\text { externa }\end{array}$ & Urgencias & IV & Sí & Sí \\
\hline 15 & 40 & Femenino & Privada & $\begin{array}{c}\text { Urgencias y } \\
\text { hospitalización }\end{array}$ & Pediatría & IV & Sí & Sí \\
\hline 16 & 64 & Masculino & Privada & $\begin{array}{c}\text { Urgencias y } \\
\text { hospitalización }\end{array}$ & Neumología & III & Sí & Sí \\
\hline 17 & 69 & Femenino & Privada & $\begin{array}{l}\text { Consulta } \\
\text { externa }\end{array}$ & Endocrinología & IV & Sí & No \\
\hline
\end{tabular}

Fuente: elaboración propia

en el proceso; esta dinámica confrontó de modo permanente el punto de vista de los investigadores ${ }^{(11)}$.

Con un trato respetuoso los investigadores nos presentamos ante los participantes como académicos interesados en comprender su experiencia sin prometer nada a cambio, a cada entrevistado se le dio a conocer los objetivos de la investigación antes de iniciar las entrevistas y el carácter voluntario de las respuestas durante el desarrollo de la misma. Esto permitió que aceptaran participar y que expresaran abiertamente sus relatos, con detalles de lo que representó la experiencia expresando los sentimientos asociados con ella.

El grupo investigador hizo conscientes los constructos de la partida inicial y tomó decisiones de forma flexible, 
reconociendo las limitaciones ${ }^{(12)}$, de forma transparente, entre las cuales se puede mencionar las propias preconcepciones de lo ético en las atenciones en salud por la experiencia clínica de algunos de los investigadores, lo que llevó a una emergencia teórica heterogénea.

\section{RESULTADOS}

Entre los significados que emergieron de los discursos de los participantes se encontró que el de mayor fuerza fue el sentirse como un asunto técnico instrumental en el que el paciente entiende que "hablan de mí”. Este significado central está en relación con otros que se analizaron en cuatro subcategorías enmarcadas en la matriz paradigmática diseñada por los autores, en la cual se observa como principal fenómeno el sentirse convertido en un asunto técnico y tener que tomar distancia de su propia situación.

Como causas de sentirse instrumento de aprendizaje, surgieron la sobrevaloración del interés docente sobre el asistencial y la dedicación de tiempo mayor hacia el estudiante comparado con la del paciente. Como relaciones de acción e interacción se evidenciaron asuntos relacionados con la comunicación y el respeto, el número de estudiantes que los pacientes estaban dispuestos a aceptar en la consulta, el respeto a su intimidad y el consentimiento previo. Las consecuencias finales dieron cuenta de una predisposición variable de los pacientes hacia ese tipo de atención según otras experiencias previas, soportada en el espíritu de servicio para el aprendizaje de otros, hasta la desconfianza por la posible vulneración de la confidencialidad (Figura 1).

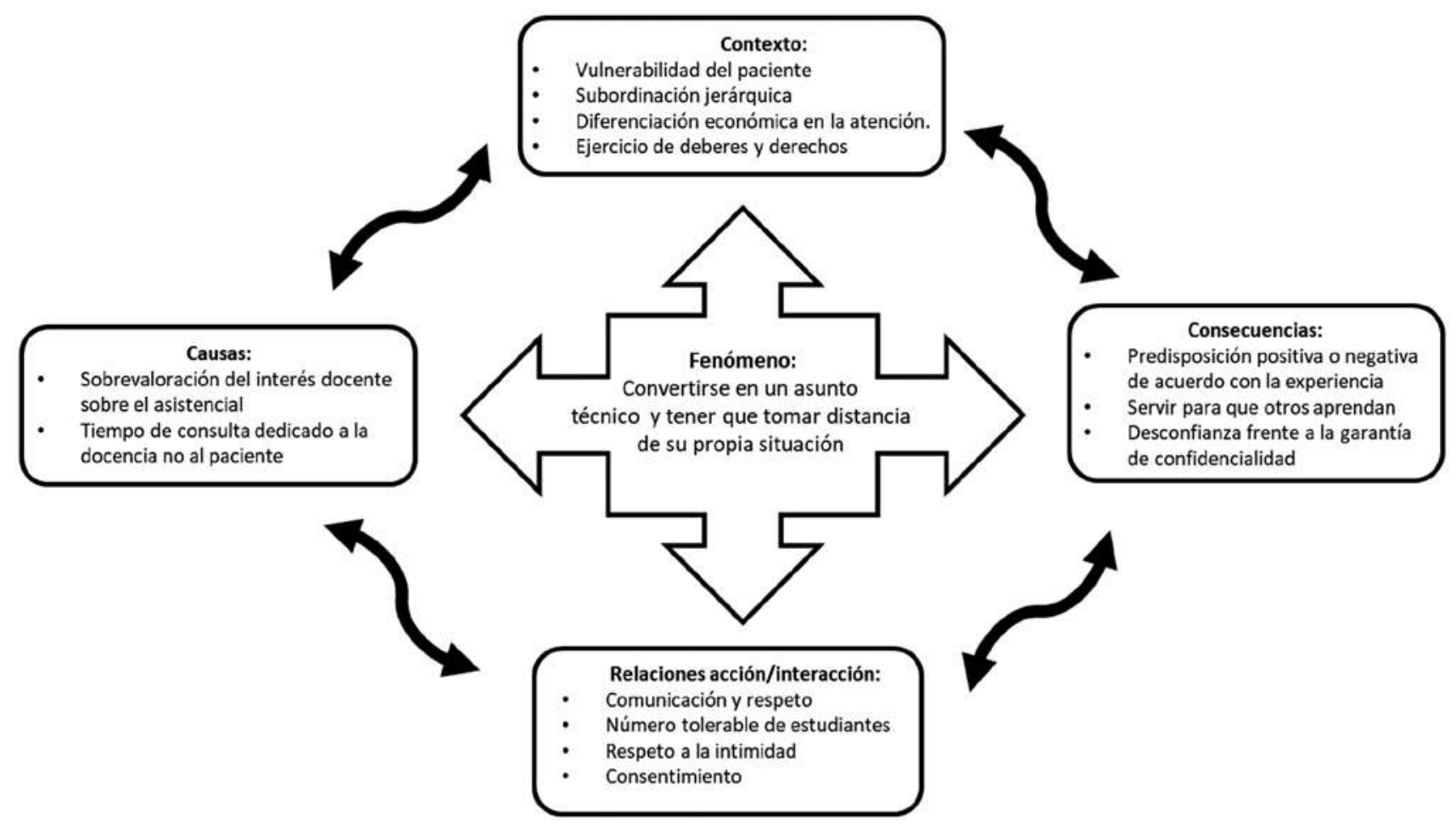

Figura 1. Matriz paradigmática. Significados de bioética que emergen en los pacientes participantes en los procesos de formación médica. Fuente: adaptación de los autores de la propuesta de Strauss y Corbin, en bases de la teoría fundada 


\section{Fenómeno}

En los procesos de atención de salud en el marco de dinámicas de formación médica surge la subvaloración y cosificación como siognificado que puede darse en los pacientes. Unos de los fenómenos emergentes son la emoción y el sentimiento que se generan en los pacientes cuando se habla de ellos a los estudiantes sobre los eventos clínicos que aquellos presentan. Esos significados expresan la sensación de convertirse en un asunto técnico, con un lenguaje incomprensible que genera sensaciones de extrañeza y distanciamiento de su propia situación. Esto se produce en el paciente cuando se siente subvalorado y marginado de una explicación sobre su proceso de atención médica. Escucha que hablan de él en términos que lo reducen a un suceso clínico y biológico, independiente de la historia que como ser humano representa, sin tener en cuenta la escucha atenta de quien quiere entender cuál es su situación y sus posibilidades.

“...es como si uno fuera el animal de estudio ahí, como el que no puede hablar, no le hablemos, se sentía maluco, como si no se pudieran comunicar con uno, ser amables, ser humanos." E03:03

"A mí no me gusta, porque igual ahí sí todos opinan y comparten las ideas entre los estudiantes; uno como paciente no le dice nada a ellos, pero entre ellos, sí hablan sobre lo que uno posiblemente pueda tener, entonces, a mí no me gustó." E1:06.

\section{Causas}

La sobrevaloración del interés docente sobre el asistencial se incrementa cuando lo que prima es el objetivo docente, en consecuencia la atención del paciente pasa a ser solamente instrumental. Este fenómeno se acentúa con expresiones que olvidan al paciente como sujeto activo que espera participar en los procesos que tienen que ver con su salud y su vida. Se resalta la ética y el trato humano como aspectos fundamentales en la formación de los médicos, pero al tiempo se expresa el menoscabo de estos fundamentos que afectan a los pacientes. En los discursos es reiterativa la inconformidad con respecto al tiempo dedicado al ingreso de datos al sistema de información y aspectos administrativos, lo que perciben como falta de interés en la consulta y en el discurso del paciente. “...como te digo, la disponibilidad del médico para hablar con uno o hacerle cualquier pregunta era muy poca y menos cuando estaba con los estudiantes..." E3:04.

“Tal vez lo más maluco, [...] es que cuando uno va a consulta, ellos son con el tiempo muy restringido y como muy metidos en hacer, pues en vaciar en el sistema una cantidad de datos y eso les roba mucho rato del que yo considero debería ser tiempo para intercambiar con uno." E7:08.

\section{Contexto}

Entre los significados que surgieron está la vulnerabilidad del paciente, quien llega a la consulta con la perspectiva de entregar un problema personal que el médico debe ayudarle a solucionar. Existe la expectativa de encontrar en la atención médica unas condiciones que generen confianza con un trato personalizado y respetuoso.

El menoscabo del reconocimiento integral del paciente lleva a que este se sienta vulnerado, lo que le genera mayor incertidumbre, indefensión, sufrimiento y subordinación que lo desvincula de su capacidad de decidir sobre sí mismo. Se expresó una especial insatisfacción cuando percibe que el especialista delega responsabilidades sin explicaciones claras al respecto y el paciente nota su ausencia o descuido en el cumplimiento de su cita.

En los sionificados surge la percepción de jerarquías acompañadas de maltrato y verticalidad de algunos docentes, especialmente con estudiantes de posorado, lo que lleva a los pacientes a identificarse con estos en su condición de subordinación y asimetría, así como la diferenciación en la calidad de la atención cuando el pago es a través de pólizas de salud.

Lo anteriormente expuesto se da en un contexto social en el que las personas cada vez más reconocen sus derechos y deberes en la atención de salud. En este estudio se encontró una limitación importante en la identificación de ambos, con énfasis en la exigencia de los derechos $y$ menor reconocimiento de los deberes.

“...uno hospitalizado se siente muy mal, uno es como [si] uno estuviera en la prisión, entonces uno ahí como en un estado de indefensión muy 
tenaz y no encuentra como un interlocutor para hablar de eso, muy complicado." E07:04.

“...que [el médico] me trate [...], como con una actitud, como una amiga, como una persona especial, no como un paciente ...... Que a ver qué tiene y ya, si no que yo sienta que él me trata bien." E8:02.

\section{Relaciones de acción e interacción}

La comunicación surge como el elemento esencial en las interacciones clínicas y se hace más compleja con la presencia de estudiantes. En la atención que hace parte de las prácticas académicas se produce en los pacientes una especial sensibilidad ante frases, gestos $y$ actitudes que pueden suscitar desconfianza, temor por la posibilidad de que surjan errores médicos por hacer parte de procesos de aprendizaje en los que la comunicación pudiera no ser la adecuada. Hay aceptación de estudiantes en la consulta por parte de los pacientes en el proceso de atención, siempre que se limite el número, que esté presente un docente y que este se encargue de la asistencia, con acompañamiento permanente, sin delegar funciones.

Se encuentran siognificados singulares en áreas específicas de la atención médica como la ginecológica que inciden en el respeto a la intimidad de las pacientes, quienes manifiestan no estar preparadas para la presencia de los estudiantes en este tipo de consultas.

Algunos participantes expresaron que no hubo consentimiento sobre la presencia de los estudiantes en la consulta. Cuando ellos tienen la oportunidad de consentir esa participación valoran en mayor grado esta atención, se perciben como parte del proceso educativo sobre la base de unos principios éticos que consideran indispensables.

"Yo no tengo problema, pues con que hayan estudiantes, pues, siempre y cuando haya como un marco de respeto, de dignidad, de trato amable, pero obviamente pues alguien a cargo." E12:05.

“...llegó una de las estudiantes que estuvo con él (médico) y me dijo: 'Ah vamos a hacer una punción lumbar al niño', y yo "un momentico es que el niño no viene a eso, y por qué punción lumbar, dónde está el consentimiento, dónde está? [...] no, un momentico, o sea, no, el niño no viene por una punción lumbar, el niño viene por una infección urinaria, ¿por qué punción lumbar?” E15:03.

\section{Consecuencias}

La predisposición a la atención con estudiantes fue variable porque, además de la instrumentalización, surgieron significados de valoración, inclusión y respeto, así como mejor comprensión de su situación al escuchar al docente cuando le explicaba al estudiante con un lenguaje que lograban entender. EI paciente participa en la consulta esperando una recompensa futura de carácter social para que el estudiante aprenda y se desempeñe bien en el ejercicio de su profesión, porque él o sus seres queridos pueden requerir de sus servicios en un futuro hipotético.

Hay desconfianza en el manejo de la confidencialidad por el alto número de estudiantes, lo perciben como una extralimitación que obstaculiza la autoestima y el bienestar. Los participantes encuentran una disposición en los estudiantes para responder sus preguntas, diferencian a los residentes como más prácticos y a los estudiantes de pregrado como más pendientes de su aprendizaje, más detallistas y cálidos en su acercamiento al paciente.

"Ah bueno, no, el derecho obviamente a la confidencialidad, que yo conociendo la parte de los estudiantes, y yo sé que eso no se cumple el $100 \%$, porque es muy común, pues, se supone que uno no debe comentar las situaciones de los pacientes en otro momento, porque la historia del paciente pertenece a él." E10:06.

"Y sí pienso..., primero el tema de la intimidad, eh, pues, es un tema muy delicado..., son más o menos 5 estudiantes, están los de pregrado, están los internos, están los residentes, entonces eso es muchísima gente en un consultorio y todos miránđolo a uno, todos examinánđolo y preguntándole." E10:07.

\section{DISCUSIÓN}

La educación médica mediante las prácticas académicas debe promover y respetar la autonomía de las personas, así como reconocer su dignidad humana, independiente de la condición singular, y no propiciar un conflicto al sobrevalorar el interés docente 
sobre el asistencial, lo que genera instrumentalización del paciente. Recordemos que un significado nuclear del estudio se enfocó en esa vulneración.

Lo anterior concuerda con una de las experiencias en un estudio sobre médicos en los dos primeros años después de su graduación ${ }^{(13)}$; una de las participantes se sintió tratada como un objeto por el docente por lo que llamó la atención al estudiante para que no repitiera esa actitud. Se trata de un proceder que afecta el respeto a la dionidad cuando una persona se instrumentaliza, como un medio, al servicio de otras, sin tener en cuenta la protección de su bienestar y sus derechos ${ }^{(14)}$.

Otras publicaciones se han referido a este conflicto ético ${ }^{(15)}$. En un estudio con adultos mayores surgió la necesidad de destinar mayor tiempo para que los estudiantes realicen el examen clínico y se mencionó la importancia de escuchar y tener paciencia ${ }^{(16)}$. El paciente en consulta con los estudiantes es vulnerado al ser desconocido como sujeto y actor de la práctica académica al inhibirse su participación activa. Esa vulnerabilidad, en vez de ser respetada y protegida, puede ser objeto de discriminaciones tal como emergió en significados hallados en este estudio. L. Feito ${ }^{(17)}$ explica que ser vulnerable se refiere a la finitud $y$ al riesgo de sufrir un daño, una herida, una privación. Implica fragilidad o una situación de amenaza o posibilidad de sufrir daño físico, emocional o moral, esta última por injusticia, desprecio, u otra afectación de nuestra identidad $^{(17)}$. Esa autora plantea al menos dos tipos de vulnerabilidad, la antropológica intrínseca al ser humano y la socio-política derivada de la pertenencia a un grupo, género, localidad, medio, condición socioeconómica, cultura o ambiente.

Otro siognificado emergente es la subordinación jerárquica entre docentes y estudiantes que devela la existencia de relaciones verticales $y$ de poder propias del paternalismo que ha impregnado las relaciones sociales entre diversos estamentos $y$ escenarios ${ }^{(18)}$. Sobre los derechos de los pacientes, un estudio en un hospital docente en la India mostró que la mayoría de sus participantes conocían sus derechos generales con variaciones por grupos: $67,7 \%$ conocía el consentimiento informado, pero solo $28 \%$ sabía sobre su derecho a la privacidad y confidencialidad ${ }^{(19)}$. Otro estudio, en cuatro hospitales docentes en la India, concluyó que los pacientes no son suficientemente conscientes de sus derechos ${ }^{(20)}$.
La inquietud por la intimidad se relacionó con el número de estudiantes y la necesidad sentida del paciente de percibir que el docente está pendiente de su cuidado, muy acorde con los resultados expuestos por Beca et al. (4). Un estudio de Brasil encontró que el $8,3 \%$ de los pacientes refiere como problema ser examinados por muchos estudiantes y el 6,1\% expresaron sentir vergüenza ${ }^{(21)}$.

Se reconocieron experiencias positivas al percibir al estudiante atento a brindar una buena atención, pero también surgieron recuerdos de vivencias anteriores de atenciones traumáticas que afectaron la valoración de otros encuentros por obstáculos en la comunicación. Además, emergió la percepción de que el sentido ético y el trato respetuoso se debilitan con el avance en la formación. Un estudio en Reino Unido, que indagó a pacientes y cuidadores sobre los médicos que empiezan su ejercicio profesional, destaca experiencias positivas con ellos y observa que los encontraron mejor formados en aspectos humanos que generaciones anteriores y resaltaron la importancia de la comunicación y el respeto en la formación ${ }^{(13)}$.

Sobre esa dimensión humanística, recordemos la reflexión de Camps ${ }^{(22)}$ sobre los valores en la profesión sanitaria, en la que destaca la ética de las virtudes como complemento de la ética de principios. Camps explica que las virtudes son disposiciones o maneras de ser y de actuar que requieren ser cultivadas, por lo que el proceso formativo se debe ocupar de inculcarlas y promoverlas, puesto que es necesario que tanto la esfera disciplinar como la ética, en tanto sabiduría práctica, se incorporen en la profesionalidad de acuerdo con las funciones de la medicina. Camps observa que el déficit en esa sabiduría práctica corresponde a un fallo educativo que da cuenta de un corporativismo que prioriza los conocimientos instrumentales y técnicos subvalorando la inculcación de la ética.

Surgieron expresiones de simpatía hacia los estudiantes por sus gestos, actitudes e interés por los pacientes, pero también se produjeron otras manifestaciones de malestar en que el paciente se sentía ionorado en la comunicación docente-estudiantes. Un estudio cuantitativo en un hospital en Brasil, encontró que la mayoría de los pacientes sintieron satisfacción en su participación en la práctica de semiología de los estudiantes de medicina porque lo consideraron importante para la formación y participarían de nuevas 
experiencias ${ }^{(23)}$. En una investigación similar en otro hospital brasileño, se encontró una percepción positiva de los pacientes al participar en prácticas de estudiantes de medicina, aunque describen que haIlaron ambivalencias ${ }^{(24)}$. Un estudio en Chile resaltó el agrado que expresaron los pacientes hospitalizados en contextos formativos ${ }^{(4)}$. La diferencia es que nuestros participantes fueron entrevistados en un ámbito extrahospitalario sobre experiencias anteriores de las que tenían buena recordación, lo que posibilita una visión más libre y crítica.

Otro estudio en Reino Unido resalta la necesidad de promover el diálogo entre los actores de la práctica, para ofrecer más respuestas a las expectativas de atención y tenerlos en cuenta en las decisiones ${ }^{(13)}$. La formación médica debe propender por generar disposiciones para la comunicación adecuada y respetuosa con los pacientes ${ }^{(13)}$, en reconocimiento de su dignidad y autonomía, así como para optimizar los logros en el autocuidado y una mayor adherencia a los tratamientos ${ }^{(25)}$.

El significado sobre las preocupaciones acerca de la confidencialidad reiteran los hallazgos de otras investigaciones ${ }^{(4)} y$ se expresó la vulneración de la intimidad, un derecho fundamental que Sánchez define como "la libertad, en el entorno inmediato del individuo, frente a la intromisión no deseada de otras personas" (18). Sánchez manifiesta que confidencialidad e intimidad están relacionadas, porque hay información que debe resguardarse por estar vinculada a la intimidad de los sujetos.

El paciente se identifica con el estudiante cuando vivencia relaciones asimétricas de subordinación jerárquica de estos con sus docentes, este hallazgo no estaba referenciado en los estudios revisados y hace referencia a la fuerza que mantiene el paternalismo en la formación médica. Por otra parte el médico amigo, como aspiración de los pacientes se reafirmó en los significados producidos. Emanuel ${ }^{(3)}$ y Gracia ${ }^{(26)}$, han analizado esta perspectiva ética en las relaciones clínicas que se contraponen a las relaciones paternalistas.

En este y en otros estudios se hacen aportes para fortalecer la formación bioética, coinciden en hacer un llamado a que la $\nabla 0 z$ de los pacientes deje de ser marginal y se considere sus necesidades de diálogo, confidencialidad, intimidad, autonomía y dignidad, reconociendo su protagonismo en los procesos formativos, su autonomía, sus expectativas y su rol en los currículos médicos. Estos resultados que exhiben relaciones despóticas entre docente y estudiante, deben llamar a cuestionar el paternalismo indebido que erosiona los fundamentos éticos actuales. Este estudio hace un aporte para comprender el entramado de roles que se desarrollan en las relaciones entre paciente, estudiante y profesor en los procesos de atención médica, en los cuales afloran tensiones éticas.

Una limitación de este trabajo es que la tercera parte de los entrevistados tenían vínculos laborales con entidades educativas formadoras de médicos, lo que puede generar un enfoque en el que prima su visión más experimentada que otras personas.

\section{CONCLUSIONES Y RECOMENDACIONES}

La dimensión bioética está relegada en la educación médica del medio analizado, se refleja en el malestar de los pacientes por una atención que profundiza su vulnerabilidad, afecta su dignidad humana, desconoce su capacidad de autonomía y amplía las condiciones de injusticia e inequidad por discriminaciones en distintas condiciones.

Las prácticas académicas en medicina bajo la concepción paternalista tienen el riesgo de cosificar a las personas, al limitar su condición humana a sionos y síntomas, omitiendo que son sujetos de derechos $y$ deberes, así como seres con un proyecto de vida. Con esa visión, se afecta la integridad de los pacientes al reproducir relaciones despóticas en las que se iognora el reconocimiento y el respeto a la alteridad con todas las diferencias que esta implica.

También se aprecian conductas ejemplares del trato por los estudiantes y profesores con los pacientes, respetuosos de los principios bioéticos y con disposiciones morales para la compasión, el respeto y la responsabilidad. Lo anterior debería ser objeto de análisis, para darle fuerza en la formación, en el compromiso con el juramento hipocrático, actualizado en el año 2017 por la Asociación Médica Mundial (27). Se incluyeron principios que ha desarrollado la bioética en las últimas décadas, como el respeto por la autonomía y la dignidad de los pacientes y el respeto a los maestros, colegas y estudiantes. 
Recordemos a Kant en una de las formulaciones de su imperativo categórico: "Obra de tal modo que uses la humanidad, tanto en tu persona como en la persona de cualquier otro, siempre como un fin al mismo tiempo y nunca solamente como un medio" (28).

El significado central que surogió en este estudio con la instrumentalización del paciente, debe ser superado a la luz de esa idea kantiana, para integrar en la educación médica un trato respetuoso basado en los principios que reconozcan la dignidad de los pacientes. De ahí que, los currículos de formación médica deben incluir el proceso de consentimiento informado para la atención con estudiantes, como lo han recomendado diversos artículos ${ }^{(4,21-24,29,30)}$, basado en una comunicación clara y comprensiva, lo que incide en el resultado de la atención como fuente de credibilidad y confianza.

Lo anterior, complementa la disposición positiva de Ios pacientes para ser atendidos como parte del proceso formativo de los médicos, sobre la base de una educación médica que debe promover los derechos $y$ deberes de los pacientes con una formación bioética en valores, principios y virtudes como esencia del profesionalismo médico.

La fascinación con la biotecnología lleva fácilmente a iognorar los espacios requeridos de formación ética y bioética, en el afán de abarcar innumerables desarrollos e innovaciones de la biomedicina. Así, pierde sentido el proceso educativo con respecto a los fines de la medicina si no se asume el reconocimiento de la alteridad y la dignidad de los pacientes, el respeto a su vulnerabilidad y autonomía, así como la debida protección cuando estas se encuentran limitadas y en peligro. Gilbert Hottois (31) plantea que la bioética ofrece una vía intermedia entre la tecnofilia y la tecnofobia para regular la ambigüedad entre beneficios y perjuicios a los que puede conducir el impacto de la tecnociencia.

Es fundamental, en pro del bienestar de los pacientes, de su protección y defensa de sus derechos, que la educación médica incorpore tanto los conocimientos biomédicos como la sabiduría práctica de la ética y la bioética en el abordaje de la vulnerabilidad en el proceso salud-enfermedad. La Declaración de Bioética y Derechos Humanos ${ }^{(32)}$ incluye el principio de Responsabilidad Social y Salud, en el que explica: "el goce del grado máximo de salud que se pueda lograr es uno de los derechos fundamentales de todo ser humano sin distinción de raza, religión, ideología política o condición económica o social". Se indica que los principios "deben entenderse como complementarios y relacionados unos con otros".

Es necesario garantizar en el currículo médico un espacio de análisis, conceptualización y reflexión bioética aplicada a la relación con los pacientes, abarcando conocimientos, actitudes y comportamientos ${ }^{(33)}$ para el desarrollo profesional. Esto entraña permear la educación médica, con trabajo interdisciplinario soportado en unidades institucionalizadas dedicadas a la bioética en cada facultad, encargadas, entre otras funciones, de hacer seguimiento a la voz de los pacientes. Se recomienda, especialmente, un nivel mínimo de formación bioética de los docentes, con un desarrollo continuo, puesto que son ejemplos con fuerte influencia sobre el modelaje de los estudiantes. La literatura académica ha insistido en ello ${ }^{(33)}$, pero aún se observa que es una tarea pendiente de asumir en muchos de los programas de medicina.

\section{AGRADECIMIENTOS}

Los autores agradecemos a los pacientes que nos aportaron su experiencia, a los estudiantes de medicina Anderson Parra Hernández y Dayana Vanessa Montoya Herrera quienes aportaron la revisión bibliográfica inicial.

\section{CONFLICTOS DE INTERESES}

Ninguno por declarar.

\section{REFERENCIAS BIBLIOGRÁFICAS}

1. Lavados Montes C, Lolas Stepke F, Drumond de Frías JG. Bioética. Acta bioeth. 2013;19(1):157-61. DOI 10.4067/S1726-569X2013000100020.

2. Collado J. La bioética como ciencia transdisciplinar de la complejidad: una introducción coevolutiva desde la gran historia. Rev Colomb Bioet. 2016;11(1):54-67.

3. Emanuel EJ, Emanuel L. Cuatro modelos de Ia relación médico paciente. Madrid: Bioética para clínicos; 1999. 
4. Beca JP, Browne F, Valdebenito C, Bataszew A, Martínez MJ. Student patient relationship from the patient's point of view. Rev Med Chil. 2006;134(8):955-9. DOI / S0034-98872006000800002.

5. Sánchez MA. El humanismo y la enseñanza de las humanidades médicas. Educ Med. 2017;18(3):212-8. DOI 10.1016/j.edumed.2017.03.001

6. Molina Montoya NP. Formación bioética en ciencias de la salud. Cienc Tecnol Salud Vis Ocul. 2016;14(1):117-32. DOI 10.19052/sv.3544.

7. Arcila PA, Mendoza YL, Jaramillo JM, Cañón OE. Comprensión del significado desde Vygotsky, Bruner y Gergen. Diversitas; 2010; 6(1):37-49.

8. Lincoln YS, Lynham SA, Guba EG. Paradigmatic controversies, contradictions and emerging confluences, revisited. Los Angeles: Sage; 2011.

9. Corbin J, Strauss A. Francis K. Basics of qualitative research techniques and procedures for developing grounded theory. California: Sage Publication; 2006.

10. Blumer H. The Metodological Position of Symbolic Interactionism. Vilnius University Press. 1969;2(2).

11. Yeasmin S, Rahman KF. "Triangulation” research method as the tool of social science research. BuP Journal. 2012;1(1):154-63.

12. Engward H, Davis G. Being reflexive in qualitative grounded theory: Discussion and application of a model of reflexivity. J Adv Nurs. 2015;71(7):1530-8. DOI 10.1111/jan.12653.

13. Kostor C, Rees C, Gormley G, Monrouxe L. 'I did try and point out about his dignity': a qualitative narrative study of patients and carers' experiences and expectations of junior doctors. BMJ Open. 2018;8(1):e017738. DOI 10.1136/bmjopen-2017-017738.

14. Soberanes JL. Reflexiones sobre el concepto de dignidad humana y su proyección en el campo de la bioética. En: Gonzáles Valenzuela J, coordinador. Perspectivas de bioética. México: UNAM, Facultad de Filosofía y Letras; 2007. p. 237-324.

15. Hicks LK, Lin Y, Robertson DW, Robinson DL, Woodrow SI. Understanding the clinical dilemmas that shape medical students' ethical development: questionnaire survey and focus group study. BMJ. 2001;322(7288Re):709-10.

16. Aquilina T, Thompson S, Metcalfe K, Hughes H, Sinclair L, Batt F. The involvement of older inpatients in medical student education. Eur Geriatr Med. 2018; 9(1):77-82. DOI 10.1007/s41999-017-0023-1.

17. Feito L. Vulnerabilidad. An. Sist. Sanit. Navar. 2007;30(3):7-22.

18. Sánchez Gonzalez MA. La relación clínica. Paternalismo y autonomía. Objeción de consciencia. En: Bioética en Ciencias de la Salud. Barcelona; Masson 2012. p. 189-98.

19. Agrawal U, D'Souza BC, Seetharam AM. Awareness of Patients' Rights among Inpatients of a Tertiary Care Teaching Hospital- A Cross-sectional Study. J Clin Diagn Res. 2017;11(9):IC01-IC06. DOI 10.7860/ JCDR/2017/24961.10544.

20. Yaghobian M, Kaheni S, Danesh M, Rezayi Abhari F. Association between Awareness of Patient Rights and Patient's Education, Seeing Bill, and Age: A CrossSectional Study. Glob J Health Sci. 2014;6(3):55-64. DOI 10.5539/gjhs.v6n3p55.

21. Silva Junior G, Duarte R, Menezes A, Morais A, Alves A, Daher E. Percepção dos pacientes sobre aulas práticas de medicina: uma outra ausculta. Rev Bras Educ Med. 2014;38(3):381-7.

22. Camps $\nabla$. Los valores éticos de la profesión sanitaria. Educación Médica. 2015;16(1):3-8. DOI 10.1016/j.edumed. 2015.04.001.

23. Ramalho A, Da Silva MR. Percepção dos pacientes sobre a sua participação como Instrumento de Aprendizado nas aulas práticas de Semiologia. ACM. 2006;35(3):35-41.

24. Sousa-Muñoz R, Ronconi D, Ramalho C, Andrade M, Silva A, Pereira G et al. Opinión de los pacientes sobre su participación en la enseñanza práctica de medicina en un hospital universitario de Brasil. Educ méd. 2011;14(1):35-7.

25. Hopkins J. Defining the Patient-Physician Relationship for the 21st Century. Dis Manag. 2004;7(3):161-79. DOI 10.1089/dis.2004.7.161.

26. Gracia D. Epilogo: el médico perfecto. En: Fundamentos de Bioética. Madrid: Triascastela; 2008.p. 597-602.

27. World Medical Association [Internet]. WMA - The World Medical Association-WMA Declaration of Geneva; 2008. [Accedido 26 Nov. 2018]. Disponible en: https://www. wma.net/policies-post/wma-declaration-of-geneva/

28. Kant M. Fundamentación de la metafísica de las costumbres [Internet]. Traducido por Manuel García Morente. San Juan, Puerto Rico: edición de Pedro M. Rosario Barbosa, 2007. [Acceso el 14 de noviembre 
de 2018] Disponible en: https://pmrb.net/books/kantfund/fund_metaf_costumbres_vD.pdf

29. Dearman S, Joiner A, Gordon M, Vince G. Experiences of Patients with Mental Illness' Interactions with Medical Students: A Systematic Review. Can J Psychiatry. 2017;63(1):4-11. DOI 10.1177/0706743717730824.

30. Howe A, Anderson J. Involving patients in medical education. BMJ. 2003;327(7410):326-8. DOI 10.1136/ bmj.327.7410.326.
31. Hottois G. El paradigma bioético. Una ética para la tecnociencia. Barcelona: Anthropos; 1991.

32. Unesco. Declaración universal de Bioética y Derechos Humanos [Internet]. UNESCO; 2005 [Consultado 2018 nov 14] Disponible en: https://bit.ly/19wMRYn

33. León Correa FJ. Enseñar Bioética: cómo transmitir conocimientos, actitudes y valores. Acta Bioéthica. 2008,14(1):11-18. DOI 10.4067/S1726569X2008000100002. 